\title{
Coffee and doughnut maculopathy: a cause of acute central ring scotomas
}

\author{
John B Kerrison, Stephen C Pollock, Valerie Biousse, Nancy J Newman
}

Department of Ophthalmology, Emory University School of Medicine, Atlanta, Georgia, USA J B Kerrison

V Biousse

N J Newman

Department of Neurology

V Biousse

N J Newman

Department of Neurological Surgery N J Newman

Department of Ophthalmology, Duke University School of Medicine, Durham, North Carolina, USA S C Pollock

Correspondence to: Nancy J Newman, MD, Neuro-ophthalmology Unit, Emory Eye Center, 1365-B Clifton Rd NE, Atlanta, GA 30322, USA

Accepted for publication 9 September 1999

\begin{abstract}
Aims-To report the clinical features of five patients with non-progressive central ring scotomas of acute onset associated with excellent retained visual acuity.

Methods-Complete neuro-ophthalmological examinations were performed. Visual fields were performed by tangent screen, Goldmann, or Humphrey perimetry. In some cases further testing was carried out including fundus photography, fluorescein angiography, ERG, VEP, and neuroimaging.

Results-The patients were three women and two men whose ages ranged from 25 to 57 years. Four patients were heavy caffeine consumers while the fifth patient experienced an episode of hypotension. Vision loss was acute in all cases. The onset of vision loss was bilateral/simultaneous in three cases, bilateral/sequential in one case, and unilateral in one case. All affected eyes retained visual acuities of $20 / 25$ or better. Colour vision was subnormal in three of four cases. Visual field defects were characterised by a central ring scotoma having an outer diameter less than 10 degrees. Fundus examination demonstrated temporal optic nerve pallor in three patients (five of 10 affected eyes) and reddish, petaloid macular lesions in one patient. Good visual acuity was maintained for the duration of follow up in all five patients.

Conclusion-Central ring scotomas with excellent retained visual acuity may present as an acute, bilateral syndrome in patients who are heavy caffeine consumers. The configuration of visual field loss and its location, combined with the presence of temporal pallor in five eyes, suggest that the defect localises to the inner layers of the macula. While these cases could be considered an expansion of the clinical spectrum of acute macular neuroretinopathy, some may represent a distinct entity.

(Br F Ophthalmol 2000;84:158-164)
\end{abstract}

Ring scotomas are annular visual field defects centred on fixation. The ring may be midperipheral, in which case the annulus passes between 30 and 60 degrees; pericentral, in which case the ring incorporates the physiological blind spot; or central. The central ring scotomas generally reside within the central 10 degrees. Mid-peripheral ring scotomas are a feature of retinitis pigmentosa ${ }^{1}$ and aphakic correction. ${ }^{2}$ Pericentral ring scotomas occur in both retinal ${ }^{3-6}$ and optic nerve diseases. ${ }^{7}$ Disorders associated with central ring scotomas have not been well characterised. This report describes five patients who had the acute onset of ring scotomas within the central 10 degrees with retention of good visual acuity.

\section{Methods}

Patients underwent complete neuro-ophthalmological examination. Colour vision was assessed using the 14 numbered Ishihara plates (after the control plate) or using the first 10 Hardy-Rand-Ritter (HRR) plates. Pupils were assessed using a transilluminator light, and relative afferent pupillary defects were quantified with neutral density filters. Static Humphrey perimetry was performed using the $24-2$ and 10-2 programs. Tangent screen testing was performed in two cases and kinetic Goldmann perimetry in one case. Fluorescein angiography was obtained in all patients. Full field electroretinograms (ERGs) were recorded using the International Society for Clinical Electrophysiology of Vision standard techniques under scotopic and photopic conditions.

\section{Results (Table 1; Fig 1)}

Vision loss was acute in all patients. The onset of vision loss was bilateral/simultaneous in three cases, bilateral/sequential in one case, and unilateral in one case. Four patients were heavy caffeine consumers, drinking on the order of one litre of caffeinated soft drinks or 20 cups of coffee a day. A fifth patient had an episode of hypotension during epidural anaesthesia before vision loss. All affected eyes retained visual acuities of 20/25 or better. Colour vision was abnormal in three of four cases as assessed by Ishihara or HRR testing. In an additional case, the patient could not see the Ishihara control plate. On Amsler grids, patients described a circular defect surrounding the centre dot, sparing the central 1-2 degrees. The outer diameter of the defect was less than 10 degrees in each case. The annular configuration was particularly evident on tangent screen testing (two patients). On automated static perimetry using the 10-2 Humphrey paradigm (three patients), the defects appeared as small central scotomas. Fundus examination was remarkable for mild temporal pallor of the optic nerve in three patients (five eyes) and bilateral, reddish, wedge-shaped macular lesions in one patient. The retinal vessels were unremarkable in all eyes. Fluorescein angiography was normal in four patients but demonstrated an area of choroidal hypofluorescence in one eye of the patient who had been hypotensive. Full field ERG was normal 


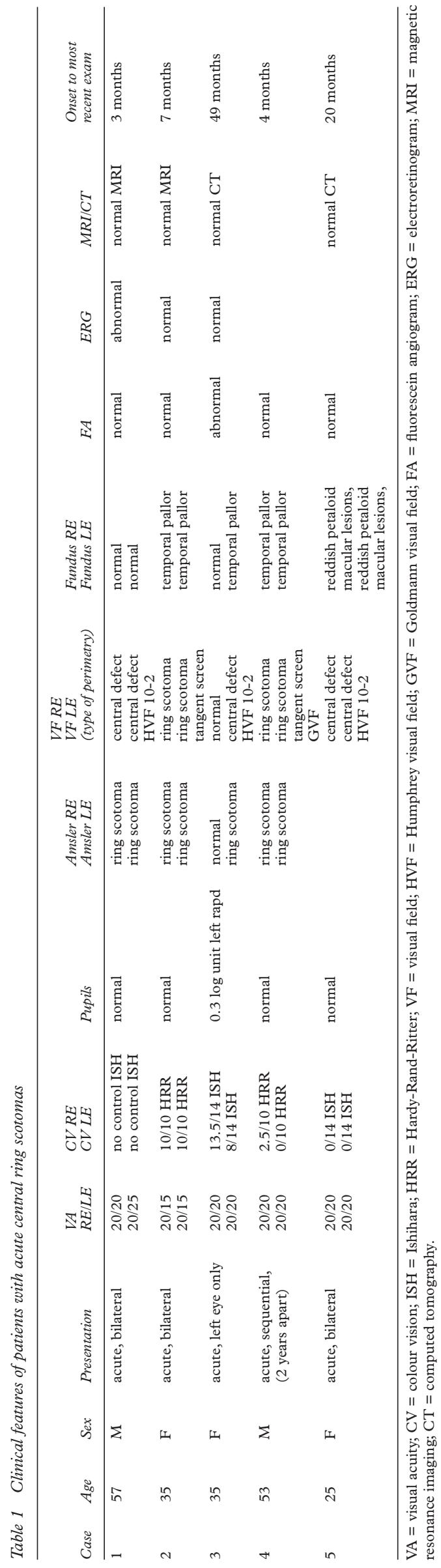

in three out of four patients. The single abnormal ERG exhibited mild prolongation of the implicit time on $30 \mathrm{~Hz}$ flicker in each eye and a small reduction in the dim white scotopic response from the right eye. Neuroimaging was normal in three cases and demonstrated non-specific periventricular foci of increased T2 signal on the MRI of one patient. Good visual acuity was maintained for the duration of follow up in all patients (range 3 months to 4 years).

\section{Case reports}

CASE 1

A 57 year old white man awoke one morning noting spots in the central vision of each eye. This impaired his reading by causing him to skip lines. The page appeared to him as if the "pen was running out of ink." On occasion, he saw "spinning fans" in the central vision of each eye. He had hypertension, treated with Lotril (amiodipine and benazepril hydrochloride, Ciba-Geigy). He consumed 10 caffeinated beverages a day. He was seen emergently by his local ophthalmologist who found a visual acuity of 20/25 in each eye, central ring scotomas on Amsler grid testing in each eye, normal pupils, and a normal fundus examination in each eye. A fluorescein angiogram showed normal macular perfusion without late leakage. An extensive laboratory evaluation was unremarkable. Magnetic resonance imaging (MRI) of the brain with contrast demonstrated mild, non-specific periventricular foci of increased T2 signal that did not enhance with contrast. Neuro-ophthalmological examination 4 months later revealed a visual acuity of 20/20-2 in the right eye and 20/25-2 in the left eye. During visual acuity testing, the patient was only able to find and identify one letter at a time. He could not see the control Ishihara testing plate with either eye. On Amsler grid testing, he described a scotomatous ring surrounding fixation in each eye (Fig 1). Humphrey visual fields were performed using a 10-2 protocol and demonstrated bilateral, incongruous central defects (Fig 1). The pupillary light reactions were normal without a relative afferent pupillary defect. Biomicroscopy was normal except for bilateral Krukenberg spindles and iris transillumination defects consistent with pigment dispersion syndrome. Intraocular pressures were normal. Dilated fundus examination was normal except for bilateral posterior vitreous detachments and mild pigmentary stippling in the maculae. An ERG was normal except for a mild prolongation of the implicit time on $30 \mathrm{~Hz}$ flicker in each eye and a small reduction in the dim white scotopic response from the right eye.

CASE 2

A 35 year old woman experienced the acute onset of simultaneous bilateral clouding of her central vision. She had a history of hypertension and cigarette smoking. She consumed a litre of caffeinated soft drinks per day. Her medications included Norvasc (amiodipine besylate, Pfizer), Reglan (metoclopramide, A H Robins), and Monopril (eosinopril 
Case 1 10-2 Humphrey visual fields Left eye

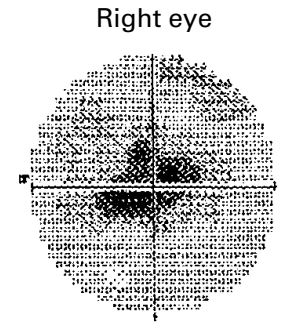

Case 2 Amsler grids

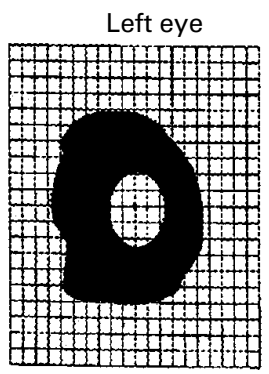

Right eye

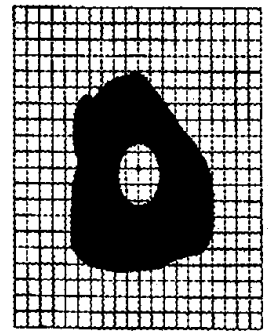

Case 3 Amsler grids

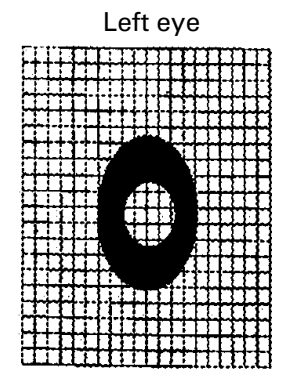

Right eye

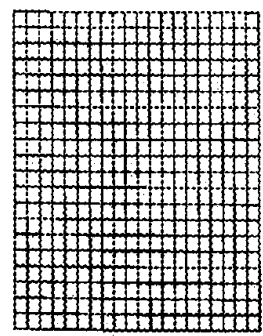

Case 1 Amsler grids

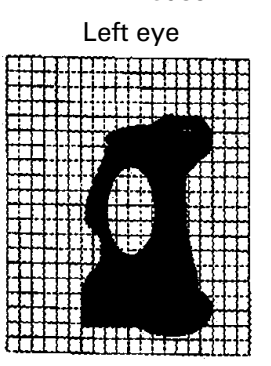

Right eye

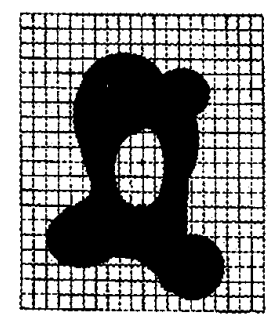

Case 3 10-2 Humphrey visual fields

Left eye

Right eye
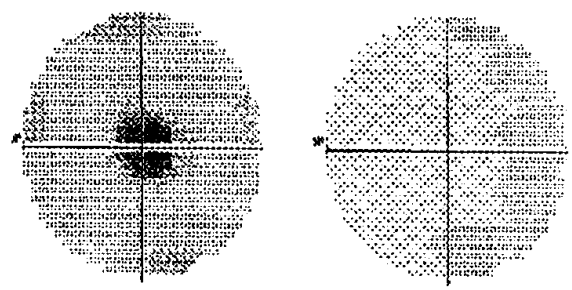

Case 4 Amsler grids

Left eye

Right eye
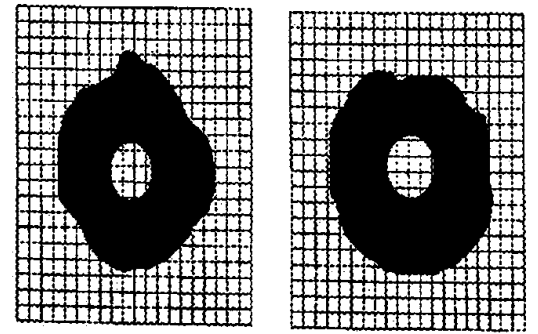

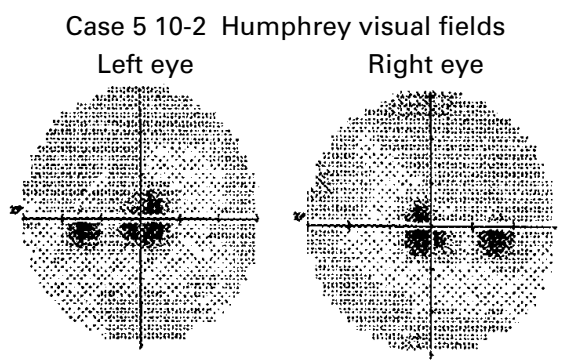

Figure 1 Central visual defects on Amsler grids and static Humphrey visual fields in patients with acute central ring scotomas.

sodium, Bristol-Myers-Squibb) Initial evaluations disclosed central visual defects in each eye. An MRI of the brain with contrast disclosed no abnormalities. A full field ERG was normal. Visual evoked potential testing was consistent with an abnormality of the anterior visual pathways. A neuroophthalmological examination performed 7 months after vision loss disclosed a visual acuity of 20/15 in each eye. Colour vision testing in each eye with HRR plates was normal. Bilateral ring scotomas were evident on Amsler grids (Fig 1) and on tangent screen testing with a red test object. The defects were located between 2 and 6 degrees from fixation. Pupillary examination, biomicroscopy of the anterior segments, and intraocular pressures were normal.
Ophthalmoscopy disclosed moderate temporal pallor of the optic nerve head, reduced nerve fibre layer reflexes in the papillomacular bundle, and blunting of the foveal reflex in each eye.

CASE 3

A 35 year old white woman had a history of myopia, central serous chorioretinopathy, obesity, hypercholesterolaemia, and toxaemia with her first pregnancy. She drank six to eight cups of coffee per day. She presented to her retina specialist with a complaint of a ring around the central dot on Amsler grid testing of the left eye. She had a normal appearing retina and fluorescein angiogram. Neuroophthalmological examination 5 months later 
disclosed a visual acuity of $20 / 20+3$ in the right eye and 20/20-2 in the left eye. Colour vision was 13.5 out of 14 Ishihara plates in the right eye and 2.5 out of 14 plates in the left eye. Amsler grid testing disclosed a ring scotoma around fixation in the left eye only (Fig 1). Humphrey visual fields using the 30-2 protocol disclosed a normal right eye and central depression in the left eye. A Humphrey 10-2 visual field in the left eye showed a central defect (foveal threshold: $37 \mathrm{~dB}$ right eye and 23 $\mathrm{dB}$ left eye) (Fig 1). Pupillary examination disclosed a 0.3 log unit left relative afferent pupillary defect. Biomicroscopy of the anterior segments and intraocular pressures were normal. Dilated fundus examination of the right eye was normal. In the left eye, mild disc pallor was present. Further testing included a normal computed tomograph (CT) scan of the orbits, a normal full field electroretinogram, and a normal laboratory evaluation, including erythrocyte sedimentation rate, rapid plasmin reagin (RPR), antinuclear antibodies (ANA), and complete blood cell count (CBC). The patient has been followed for 4 years without change in visual acuity or visual fields.

CASE 4

A 53 year old man experienced the sudden onset of a spot in the central portion of the vision in his left eye. At the centre of this spot was a clear area through which he could see. It was recommended that he take aspirin. The spot remained unchanged. Two years later, while playing golf, he noted that objects viewed with his right eye had a "misty" appearance but that there remained a small central aperture through which he could see clearly. He had a history of classic migraine headaches and took a multivitamin once a day. He was a 40 pack a year cigarette smoker and drank 20 cups of coffee a day. He consumed two alcoholic drinks three days per week. On initial evaluation by a retinal specialist soon afterwards, his visual acuity was $20 / 25$ in the right eye and $20 / 50$ in the left eye. He had bilateral, central, doughnut-shaped scotomas, no relative afferent pupillary defect, and no fundus abnormalities. On neuro-ophthalmological examination 3 months later, he had a best corrected visual acuity of 20/20 in both eyes. He correctly identified 2.5 out of $10 \mathrm{HRR}$ plates in the right eye and no plates in the left eye. On Amsler grid testing, he described a scotomatous ring surrounding central fixation in each eye (Fig 1). Tangent screen testing at a distance of 2 metres demonstrated bilateral central ring scotomas. The annulus of the ring passed between 2 and 7 degrees in the each eye, with the width being smallest along the 6 o'clock meridian. Goldmann perimetry in the right eye disclosed a bilobed defect to the I1e target within 5 degrees of fixation. A narrow tongue of preserved sensitivity extended upwards through the defect. Another scotoma to the I1e stimulus was oblong in shape and located 20 degrees superotemporal to fixation. In the left eye, a central ring scotoma was present. Pupillary examination disclosed no relative afferent pupillary defect. Biomicroscopy of the anterior segments and intraocular pressures were normal. In each eye, dilated fundus examination disclosed a cup to disc ratio of 0.7 with mild temporal pallor, decreased nerve fibre layer reflexes in the maculopapillary bundle, a diminished foveal reflex, and mild attenuation of the retinal arterioles.

CASE 5

A 25 year old white woman experienced bilateral, painless, acute loss of vision a day after spontaneous vaginal childbirth. The delivery was unremarkable except for a brief episode of hypotension during epidural anaesthesia. She underwent computed tomography (CT) of the head without contrast, lumbar puncture, visual evoked response (VER), and carotid ultrasonography, all of which yielded normal results. On neuro-ophthalmological examination 19 months later, she had a visual acuity of $20 / 20$ in each eye. She was only able to identify the Ishihara control plate with some difficulty in each eye. Humphrey visual fields performed using the 30-2 protocol disclosed bilateral central defects (foveal threshold: $36 \mathrm{~dB}$ right eye and $31 \mathrm{~dB}$ left eye) (Fig 1). Pupillary examination, biomicroscopy of the anterior segments, and intraocular pressures were normal. Dilated fundus examination disclosed bilateral reddish, petaloid macular lesions. Both optic nerves appeared healthy, as did the vessels and periphery. A fluorescein angiogram demonstrated choroidal filling defects nasal to the fovea in the right eye.

\section{Discussion}

Each of our patients experienced the sudden onset of a unilateral or bilateral central ring scotoma. The visual defect was maximal at onset and did not improve or progress. The scotomatous ring extended between 1 and 8 degrees from fixation. Because automated static perimetry tests a matrix of fixed points, the defect appeared as a central scotoma when tested by this modality (Fig 1). However, the ring configuration was evident on Amsler grid, Goldmann perimetry, and tangent screen testing and was attested to by the retention of excellent visual acuity.

The term ring scotoma may be applied to any visual field defect that encircles fixation. Morphologically, such defects can be separated into three categories: mid-peripheral ring scotomas, pericentral ring scotomas, and central ring scotomas.

Mid-peripheral ring scotomas are a characteristic clinical feature of retinitis pigmentosa. ${ }^{1}$ In this condition, scotomas initially develop between 40 and 60 degrees and then progressively coalesce. They also may gradually expand at both their peripheral and central margins. Mid-peripheral ring scotomas also occur as a consequence of aphakic spectacle correction. $^{2}$

Pericentral ring scotomas occupy the zone between 5 and 30 degrees, thus incorporating the physiological blind spot. Both retinal and optic nerve disorders may cause pericentral scotomas. 
Retinal disorders associated with pericentral ring scotomas include photoreceptor dystrophies and paraneoplastic/autoimmune disorders. Cone dystrophies that are associated with pericentral ring scotomas are typically associated with abnormalities on full field ERG and fundus examination. ${ }^{34}$ Autoimmune retinal diseases associated with ring scotomas include cancer associated retinopathy and autoimmune retinopathy. ${ }^{56}$ These disorders are characterised by progressive loss of vision, an abnormal full field ERG, and usually a normal fundus examination, although the retinal arterioles may be attenuated.

Optic neuropathies can result in pericentral ring scotomas when superior and inferior arcuate defects coalesce. Paired arcuate defects may circumferentially encompass fixation in anterior ischemic optic neuropathy, glaucoma, and optic disc drusen. ${ }^{7}$ The presence of a horizontal step, asymmetry of the ring across the horizontal meridian, and visible abnormalities of the nerve head may help to localise the disorder to the optic nerve. In a subgroup of patients with Leber's hereditary optic neuropathy, large central scotomas may fenestrate, resulting in a variant of ring scotoma and a measurable improvement in visual acuity. ${ }^{8}$

The patients that are the subject of this report have central ring scotomas, a form of ring scotomas which has not been well characterised in the literature. In 1905, Wisselink described an individual who, after a fall, had a central scotoma associated with a grey appearance of the macula. The centre of the defect cleared, leaving a small ring. ${ }^{9}$ In 1933, Fuchs described two similar cases following trauma and thought that the phenomenon represented injury to Henle's nerve fibre layer. ${ }^{10}$ Of 20 cases described by Stoll in 1950 as having "narrow ring scotomas," five patients had visual acuities of $20 / 25$ or better. Two of his cases had experienced trauma, but in three patients, he attributed the defects to "a failure of adequate supply in the small terminal retinal vessels" or "central angiospastic retinopathy," a term he attributed to Gifford and Marquardt. ${ }^{11}$

Central ring scotomas also have been described in the setting of acute macular neuroretinopathy (AMNR) where they are presumed to result from a coalescence of paracentral scotomas. ${ }^{12-14}$ AMNR is a disorder characterised by acute, usually bilateral, vision loss associated with reddish-brown macular lesions. ${ }^{12-23}$ The disease has a strong predilection for young women. ERG and electrooculography are typically normal. ${ }^{15}{ }^{19}$ Fluorescein angiography is usually normal but has been reported to show subtle dilatation of macular capillaries, ${ }^{15}$ faint hyperfluorescence of the fovea in the early phases, ${ }^{13}$ and subtle areas of hypofluorescence corresponding to the reddish macular lesions at later stages. ${ }^{13} 17$ The level at which the injury occurs within the retina has not been established. Acutely, the macula appears oedematous, suggesting inner retinal involvement. ${ }^{12}{ }^{13}$ However, the documentation of an abnormality of the early receptor potential in one case suggested an outer retinal process. ${ }^{18}$ Interpretation of the level of involvement based on the biomicroscopic appearance of the lesions has varied considerably. ${ }^{1315} 17$

The cause of AMNR is not known, but both inflammatory ${ }^{19-21}$ and vasculopathic ${ }^{12} 1424$ mechanisms have been proposed. In support of an inflammatory process is the observation that most cases occur in young women and that many affected individuals report having had an antecedent viral illness. ${ }^{15-17} 1920$ Other cases occurred in the setting of allergic drug reactions. ${ }^{12}{ }^{13}$ The development of lesions characteristic of multiple evanescent white dot syndrome (MEWDS), either simultaneously or years after the appearance of macular lesions characteristic of $\mathrm{AMNR}^{21}$ has prompted the inclusion of AMNR among the inflammatory disorders grouped together as acute zonal occult outer retinopathy (AZOOR). ${ }^{24}{ }^{25}$ Nevertheless, some cases have been reported to occur in the setting of severe blood loss and moderately severe bodily injury, suggesting that focal hypoperfusion, adrenergic stimulation, or acute elevation of ocular venous pressure may represent contributing factors. ${ }^{12} 23$

One could argue that our patients may represent a clinical variant of AMNR. Features that our patients share with AMNR patients include acute onset, bilaterality in some cases, good visual acuity, and normal fluorescein angiography. Furthermore, case 5 had retinal lesions typical of AMNR. By contrast, the remainder of our cases did not have the typical AMNR maculopathy. These cases are further distinguished by their older age (cases 1, 4), occurrence in men (cases 1,4 ), dyschromatopsia, and optic disc pallor (cases 2, 3, 4). The last two findings have not been reported in AMNR. Finally, while true central ring scotomas have been described in AMNR, they appear to be infrequent, occurring only in the setting of intravenous sympathomimetic administration. ${ }^{12-14}$ More commonly, the defects are paracentral, sometimes coalescing, but rarely completely encircling fixation. All of our patients had central ring scotomas. We therefore believe that the cases selected for inclusion in this study, with the possible exception of case 5, comprise a distinct clinical group and may result from a common pathophysiological mechanism.

The inner layers of the macula represent the most likely site of visual pathway injury in our patients. The observation of disc pallor in three of our patients is indicative of loss of retinal ganglion cells with anterograde (ascending) degeneration of axons. While temporal disc pallor can be a feature of cone dystrophies, presumably as a result of transynaptic degeneration, ${ }^{26}{ }^{27}$ the development of disc pallor soon after vision loss along with the generally normal ERG findings argues against an outer retinal process in our patients. The possibility that the temporal disc pallor observed in three of these patients resulted from injury to the optic nerve is likewise untenable. For an optic neuropathy to produce a central ring scotoma, those axons originating from ganglion cells that subserve vision between 2 and 8 degrees would have to be selectively lost while axons from 
ganglion cells whose receptive fields subserve the central one to two degrees of vision survive. Given the compact relation of these two subpopulations of axons within the optic nerve and the fact that they intermingle with one another, it seems unlikely that a pathological process at this location could be characterised by this degree of selectivity. Furthermore, the fact that superotemporal and inferotemporal macular projections are separated in space within the optic nerve ${ }^{28}$ means that for a hypothetical neuropathic process to be associated with a central ring scotoma having continuous boundaries, the area of neural injury would have to be perfectly symmetrical across the horizontal midline of the disc.

We believe that the pathophysiological mechanism of macular injury in our patients is transient ischaemia, based on the acute onset of symptoms, the stability of the visual deficits following onset, and the normal results of fluorescein angiography in all cases but one. The area of the macula corresponding to the central ring scotomas is characterised by a high concentration of ganglion cells and metabolic activity, features which may make this portion of the macula particularly vulnerable to the effects of ischaemia and hypoxia.

The dual circulation of the macula provides a plausible explanation for the spared aperture in patients with central ring scotomas due to vascular compromise. The outer retina and central portion of the fovea are supplied by the choroidal circulation via the choriocapillaris while the remainder of the inner retina is supplied by the retinal vessels. In patients with ischemic macular damage from reduced blood flow in the retinal microvasculature, the choroidal circulation would spare not only photoreceptors but also the ganglion cell bodies on the bank of the fovea that subserve the central one to two degrees of vision. Of course, axons of those surviving ganglion cells would still have to traverse the surrounding ischaemic zone as part of the retinal nerve fibre layer. However, as has been hypothesised by Rizzo (Rizzo JF III, unpublished data, 1993), ganglion cell axons may be more resistant to ischaemia than ganglion cell bodies, allowing the axons of the more central foveal ganglion cells to remain intact and functional despite exposure to a degree of ischaemia sufficient to destroy surrounding ganglion cells.

A discrete precipitating event was identified in only one of our patients, a young woman who experienced an episode of postpartum hypotension shortly before the onset of visual symptoms (case 5). This case also differed from the other four in that AMNR-like macular lesions were present and were associated with angiographic evidence of focal choroidal hypoperfusion. It may be that a drop in blood pressure produced a global decrease in ocular perfusion that involved both retinal and choroidal circulations. If this were so, the visible macular lesions may have been related to outer retinal ischaemia.

Of note, the other four patients were heavy caffeine consumers. Indeed, case 4 consumed approximately 20 cups of coffee a day! Caffeine is an inhibitor of adenosine, the latter being a potent vasodilator of the retinal vasculature. Therefore, large amounts of caffeine can be expected to cause retinal vasoconstriction. At a dose of $200 \mathrm{mg}$ (equivalent to approximately one cup of coffee), caffeine has been shown to modestly decrease the mean velocity of leucocytes within macular capillaries, and presumably, macular blood flow. ${ }^{29}$ The effect of large doses on macular blood flow is not known. Also unknown is the extent to which the vasoactive effects of caffeine on the retinal circulation are additive to other causes of vasoconstriction (for example, nasal decongestants, smoking, hypertension, Raynaud's phenomenon, migraine, etc).

We describe five patients who acutely developed central ring scotomas associated with excellent visual acuity. Four of the patients were heavy caffeine consumers, and the fifth patient had suffered an episode of hypotension. The configuration of the visual field defect in all cases and the observation of optic nerve pallor in three cases suggest a process localised to the inner retina. Transient ischaemia of the macula, possibly related to vasoconstriction, may be the responsible pathophysiological mechanism. This combination of clinical features leads us to suggest the term "coffee and doughnut maculopathy" to describe this condition.

We thank R Mitchell Newman Jr, MD for referring patient 1. This work was supported by a grant from the Heed Foundation (JBK) and as part of an unrestricted grant to Emory Eye Center, Emory University School of Medicine, from Research to Prevent Blindness, Inc, New York, and National Institute of Health CORE grant No P30-EYO 6360.

1 Weleber RG. Retinitis pigmentosa and allied disorders. In: Ryan SJ, Ogden TE, Schachat AP, Murphy RP, Glaser BM, eds. Retina. 2nd ed. St Louis: Mosby, 1994:335-466.

2 Dabezies OH Jr. Defects of vision through aphakic spectacle lenses. Ophthalmology 1979;86:352-79.

3 Ten Hove MW, Siatkowski RM, Smith JL. Foveal cone dysfunction syndrome. F Neuro-Ophthalmol 1998;18:9-14.

4 Szlyk JP, Fishman GA, Alexander KR, et al. Clinical subtypes of cone-rod dystrophy. Arch Ophthalmol 1993; 111:781-8.

5 Ohnishi Y, Ohara S, Sakamoto T, et al. Cancer associated retinopathy with retinal phlebitis. Br f Ophthalmol 1993;77: $795-8$.

6 Mizener JB, Kimura AE, Adamus G, et al. Autoimmune retinopathy in the absence of cancer. Am $\mathcal{F}$ Ophthalmol 1997;123:607-18

7 Miller NR, Newman NJ. Topical diagnosis of lesions in the visual sensory pathway. In: Miller NR, Newman NJ, eds. Walsh and Hoyt's clinical neuro-ophthalmology. 5th ed. Baltimore: Williams and Wilkins, 1998:249-52.

8 Stone EM, Newman NJ, Miller NR, et al. Visual recovery in patients with Leber's hereditary optic neuropathy and the 11778 mutation. F Clin Neuro-Ophthalmol 1992;12:10-14.

Wisselink GW. Ein Fall von traumatischer Erkrankung der Macula lutea. Klin Monatsbl Augenheilkd 1905;43:385-91.

10 Fuchs A. Uber kleinste dauernde Ringskotome nach VerkeFuchs A. Uber kleinste dauernde Ringskotome nach Verkehrsunsfällen und parazentrales Skotom nach elektrisch

1 Gifford SR, Marquardt G. Central angiospastic retinopathy. Arch Ophthalmol 1939;21:211-28. 12 O'Brien DM, Farmer SG, Kalina RE, et al. Acute macular neuroretinopathy following
ics. Retina 1989;9:281-6.

13 Guzak SV, Kalina RE, Chenworth RG. Acute macular neuroretinopathy following adverse reaction to intravenous contrast media. Retina 1983; 3:312-17.

14 Leys M, Van Slycken S, Koller J, et al. Acute macular neuroretinopathy after shock. Bull Soc Belge d' Ophtalmol 1991;241:95-104.

15 Bos PJM, Deutman AF. Acute macular neuroretinopathy. Am f Ophthalmol 1978;80:573

16 Rush JA. Acute macular neuroretinopathy. Am f Ophthalmol 1977;83:490

17 Priluck IA, Buettner H, Robertson DM. Acute macular neuroretinopathy. Am f Ophthalmol 1978; 86:775.

18 Sieving PA, Fishman GA, Salzano T, et al. Acute macular neuroretinopathy: early receptor potentials change suggests photoreceptor pathology. Br f Ophthalmol 1984:68:229-34.

19 Miller MH, Spalton DJ, Fitzke FW, et al. Acute macular neuroretinopathy. Ophthalmology 1989;96:265-9. 
20 Rait JL, O'Day J. Acute macular neuroretinopathy. Aust NZ f Ophthalmol 1987;15:337-40.

21 Gass JD, Hamed LM. Acute macular neuroretinopathy and multiple evanescent white dot syndrome occurring in the same patients. Arch Ophthalmol 1989;107:189-93.

22 Desai UR, Sudhamathi K, Natarajan S. Intravenous epinephrine and acute macular neuroretinopathy. Arch Ophthalmol 1993;111:1026-7.

23 Gillies M, Sarks J, Dunlop C, et al. Traumatic retinopathy resembling acute macular neuroretinopathy. Aust NZ F Ophthalmol 1997;25:207-10.

24 Jacobson SG, Morales DS, Sun XK, et al. Pattern of retinal dysfunction in acute zonal occult outer retinopathy. Ophthalmology. 1995;102:1187-98.
25 Gass JD. Acute zonal occult outer retinopathy. Donders Lecture: The Netherlands Ophthalmological Society, Maastricht, Holland, 19 June 1992. F Clin NeuroOphthalmol 1993;13:79-97.

26 Newman NJ, Slavin M, Newman SA. Optic disc pallor: a false localizing sign. Surv Ophthalmol 1993;37:273-82.

27 Newman NM, Stevens RA, Heckenlively JR. Nerve fiber layer loss in diseases of the outer retinal layer. $\mathrm{Br} \mathcal{F}$ Ophthalmol 1987;71:21-6.

28 Pollock SC, Miller NR. The retinal nerve fiber layer. Int Ophthalmol Clin 1986;26:201-21.

29 Lorfi K, Grunwald JE. The effect of caffeine on human macular circulation. Invest Ophthalmol Vis Sci 1991;32: 3028-32. 